\title{
El uso de Kahoot! como herramienta divertida de autoevaluación para estudiantes universitarios
}

Inigo Garcia-Rodriguez ${ }^{a}$ y Clara Pérez-Cornejo ${ }^{b}$

aUniversidad de Burgos inigogr@ubu.es, ${ }^{\text {bUniversidad de Burgos cpcornejo@ubu.es }}$

\section{\$EWWFW}

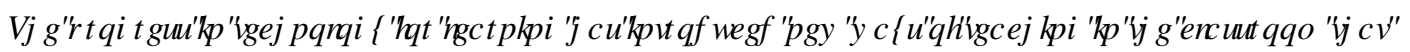

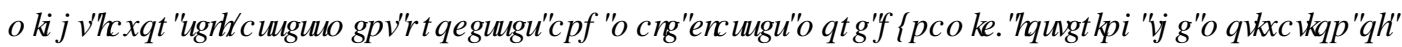

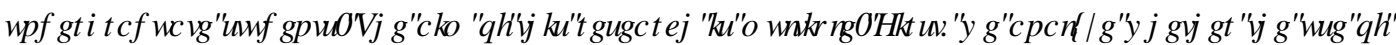

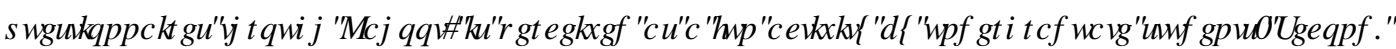

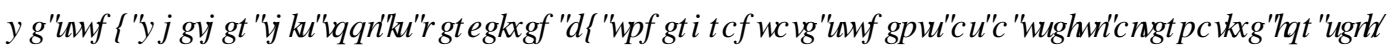

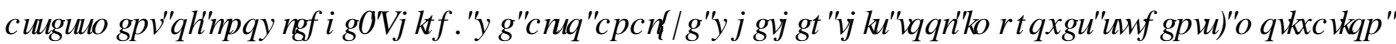

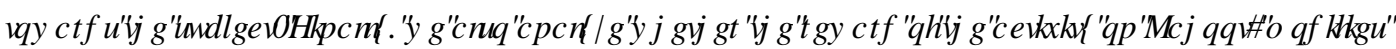

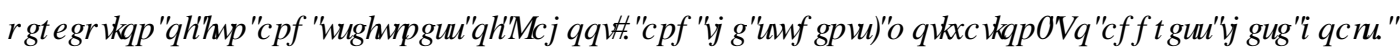

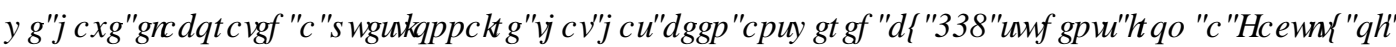

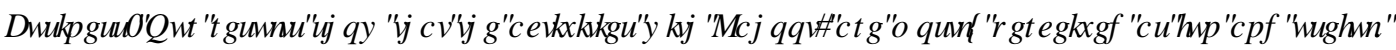

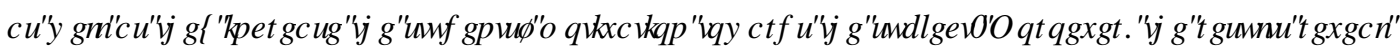

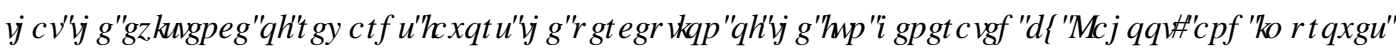
WWGHQMP RWMDFIRQ]

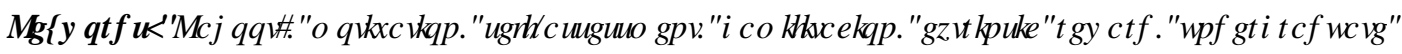
WWGHQW

\section{HXP HQ}

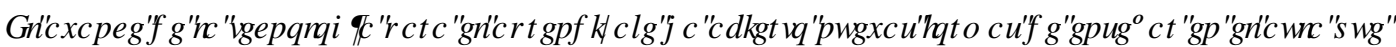

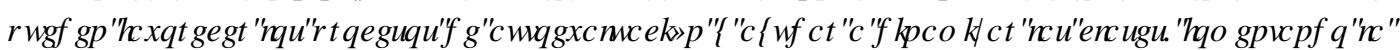

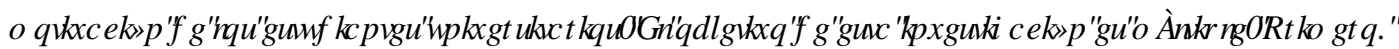
$D Q D D]$ DP RVULDUUHD] DFIY Q GHFXHMRQDURVDWDY

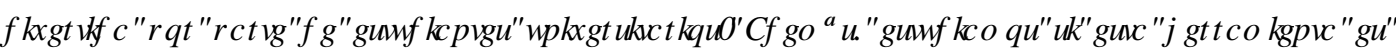

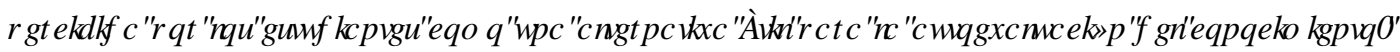

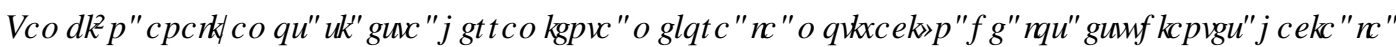

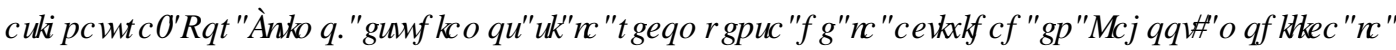

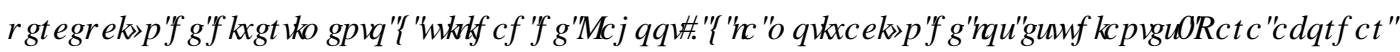

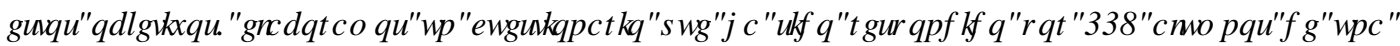
) DFXWDG GH \&IHQFWW ( FRQY PIFDV I ( P SLHDUDOMW 1 XHWRV UHXWDRV P XHWDQ TXH GDV

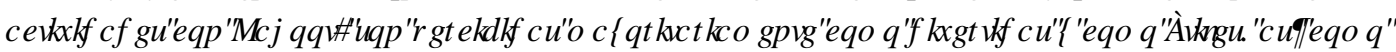

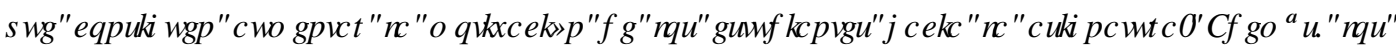

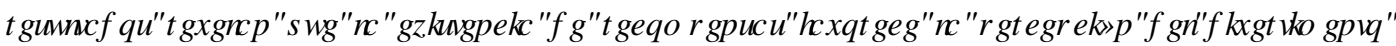

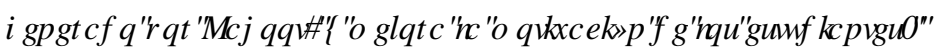

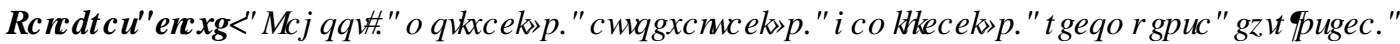
HMKGDQMALGHJWORD 


\section{Introducción}

Las nuevas tecnologías han cambiado la forma de interactuar en la docencia universitaria. No solamente sirven de vía para favorecer la accesibilidad de los materiales docentes, o la comunicación entre profesores y alumnos, sino que también ayudan a dinamizar y motivar a los alumnos en las clases a través de diferentes aplicaciones online. De hecho, la existencia de una red inalámbrica de internet en los campus universitarios y los teléfonos smartphone personales permiten contar con una infraestructura tecnológica (Wang, 2015) que facilita el empleo de aplicaciones online para la autoevaluación en prácticamente todas las aulas universitarias. Asimismo, el incremento en la utilización de los smartphone por la sociedad hace que sea necesario incorporarlos en el proceso de aprendizaje en la educación superior (Rodríguez, Restrepo y García, 2017).

Entre las aplicaciones más populares que permiten un sistema de autoevaluación dinámico en un ambiente lúdico de juego, podemos encontrar Kahoot!. Esta aplicación proporciona la experiencia de formar parte de un concurso con las características de ser un juego interactivo y competitivo Vossen (2004). Estos rasgos permiten utilizar esta herramienta para realizar cuestionarios de autoevaluación presentados de una forma dinámica como una actividad que ayude a romper con la rutina de la clase dinamizándola para favorecer la motivación del alumnado a través de dos vías. Por un lado, podríamos hablar de que favorece la motivación intrínseca de los alumnos ya que pueden tener una experiencia con ciertas dosis de diversión que anime a participar en estas actividades por el mero hecho de obtener esa experiencia. Además, por otro lado, dado que permite cierta competitividad, puede ser un instrumento que favorezca la motivación extrínseca a través de un sistema de recompensas basado en la participación del alumno de forma acertada a través de esta aplicación.

Por lo tanto, en el presente estudio queremos analizar la efectividad de Kahoot! como instrumento que ayude a la gamificación, a la autoevaluación, y a la motivación de 116 estudiantes de segundo curso de la Facultad de Ciencias Económicas y Empresariales de la Universidad de Burgos. Igualmente, también evaluaremos si el hecho de dotar a la actividad realizada con Kahoot! de una recompensa en la calificación de la asignatura tiene alguna incidencia sobre las cuestiones planteadas anteriormente.

\section{Objetivos}

Kahoot! es una aplicación online que permite transformar una clase estándar en un concurso moderado o "presentado" por el profesor, donde los concursantes son los alumnos (Wang, 2015; Wang y Tahir, 2020). Kahoot! involucra y motiva a los estudiantes a través de una experiencia de juego donde los estudiantes compiten entre ellos y que fue diseñada para crear una experiencia social en el aula (Wang, 2015) py tratar de romper con las técnicas tradicionales de aprendizaje. La dinámica funciona de la siguiente manera. El docente actúa como moderador y conecta su ordenador al proyector donde se muestran preguntas y posibles respuestas identificadas con diferentes colores. El docente lanza las preguntas de forma consecutiva que tienen una duración determinada (establecida por el docente cuando diseña el cuestionario). Estas preguntas pueden ir acompañadas de imágenes o vídeos. Por otro lado, una vez que se presenta la pregunta, tanto en la pantalla de la clase como en los dispositivos de los alumnos, aparecen las opciones de respuesta marcadas con los colores que corresponden a cada opción. Durante el período de respuesta disponible, se reproduce música que ayuda a crear una atmósfera lúdica y competitiva. Los estudiantes para tratar de lograr la máxima calificación y situarse en lo más alto del ranking de la clase, deben responder de forma rápida y correctamente en sus dispositivos spartphone. Una vez que se agota el tiempo o la pregunta ha sido respondida por todos los alumnos, el programa muestra una gráfica con las frecuencias de las diferentes opciones de respuestas que se han obtenido, al mismo tiempo que marca la opción u opciones correctas. 
Este cuadro es útil para el docente, puesto que puede ayudarle a identificar problemas de los estudiantes a la hora de asimilar ciertos conceptos de la asignatura y a detectar aquellas cuestiones que sí han sido entendidas e interiorizadas por los alumnos. Entre pregunta y pregunta, se muestra el ranking de los estudiantes que se encuentran en la parte superior de la clasificación, fomentando la competitividad entre los participantes. Además, en los dispositivos móviles se informa a los alumnos en que posición se encuentran respecto de la clase. Cuando se termina el cuestionario aparece la clasificación final. Además, esta información, sobre las posiciones y los aciertos de los alumnos, se puede descargar fácilmente de la plataforma.

Estas características de Kahoot! hacen que sea una herramienta divertida y útil que puede favorecer la motivación del estudiante en clase, y que permite de forma rápida realizar ejercicios de autoevaluación y repaso que ayuden a los alumnos a asimilar los contenidos impartidos en clase. En este sentido, los objetivos de esta investigación se centran en analizar la percepción que tienen los estudiantes sobre el nivel de divertimento y la utilidad de Kahoot!. Asimismo, también estudiamos si el empleo de esta herramienta mejora su motivación hacia la asignatura, en términos de actitud y asistencia a las sesiones. Por último, en este estudio también abordamos el papel que juega el dotar al grado de acierto en Kahoot! con una recompensa en la calificación de la asignatura, analizando la incidencia que tiene este hecho sobre las cuestiones anteriormente estudiadas (divertimento, utilidad y motivación).

\subsection{Kahoot! como herramienta para la gamificación}

La gamificación es una técnica muy útil para motivar los procesos de adquisición de conocimientos y favorecer la participación de los estudiantes en el aula (Dicheva, Dichev, Agre y Angelova, 2015; Villalustre y Del Moral, 2015). Algunos investigadores señalan que jugar puede ayudar a mantener la atención de los estudiantes y hacer que el aprendizaje sea más interesante (Boyle, 1997; Virvou, Katsionis y Manos, 2005). La gamificación del aprendizaje consiste en el uso de mecánicas de juego en entornos no lúdicos, resultando ser una metodología de aprendizaje dentro del entorno del aula (Prieto Andreu, 2020) que permite involucrar y motivar al alumnado (Kapp, 2012). Para gamificar el aprendizaje se necesita un esfuerzo que venga por parte de los docentes porque es necesaria una transformación de los materiales didácticos, adaptándolos a las nuevas experiencias y formas expresivas de la sociedad digital, cambiando el enfoque de aprendizaje basado en la producción de materiales educativos basados en la lógica de los juegos online (Prieto Andreu, 2020). Sin embargo, los estudiantes no percibirán un cambio de aprendizaje porque las nuevas tecnologías son parte del mundo de los jóvenes y son parte de su cultura (Pérez-Manzano y Almela-Baeza, 2018). Por tanto, la gamificación posibilita una experiencia interactiva, que motiva y establece un vínculo entre el alumno y el contenido que se está trabajando desde una perspectiva amigable y natural. Es por ello que en este estudio tratamos de analizar cómo los alumnos perciben la experiencia de realizar cuestionarios a través de Kahoot!. Por ello, planteamos la siguiente cuestión.

PI.1. ¿Se perciben las actividades realizadas a través de Kahoot! como divertidas y dinámicas en clase?

\subsection{Kahoot! como herramienta de autoevaluación}

La autoevaluación es una herramienta útil que ha despertado un gran interés en los investigadores a lo largo del último siglo (Falchikov y Boud, 1989). La autoevaluación permite a los estudiantes evaluar su propio desempeño y progreso en una materia. En este proceso, la integración de la tecnología en la educación ha aumentado las herramientas disponibles para probar si los estudiantes han adquirido las habilidades y los 
conocimientos enseñados en las clases. De hecho, la introducción de tecnología en el aula no es algo nuevo porque los profesores han utilizado ampliamente los sistemas de respuesta electrónica desde 1960 (Judson y Sawada, 2002). Sin embargo, herramientas en línea como Kahoot!, han democratizado esta técnica de autoevaluación, porque únicamente se necesita un ordenador conectado a una pantalla en el aula y que los estudiantes lleven sus teléfonos inteligentes al aula. Sin embargo, al estar sumergido en un ambiente de juego, tal y como proponemos en la sección previa, puede ser que los alumnos no consideren que es una actividad que les ayude a conocer si tienen ciertos niveles de conocimiento en la asignatura a través de la aplicación. Por ello, proponemos la siguiente pregunta de investigación:

PI.2. ¿Se percibe Kahoot! como una herramienta útil para la autoevaluación de los estudiantes en el aula?

\subsection{Kahoot! y motivación intrínseca y extrínseca}

La motivación en la educación está relacionada con las opiniones, juicios y valores que los estudiantes tienen sobre objetos, eventos o dominios de la materia. Igualmente, también se refiere a la opinión del estudiante sobre la eficiencia o eficacia de los métodos de aprendizaje y enseñanza (Boekaerts, 2002). Sin embargo, la fuente de motivación es compleja (Boekaerts, 2002). De forma específica, la motivación intrínseca (MI) se entiende como un signo de competencia y autodeterminación (Deci y Ryan, 1985; Deci, Vallerand, Pelletier y Ryan, 1991). Esta motivación intrínseca provoca que el alumno experimente placer y satisfacción al realizar determinadas actividades, ya sea por el desempeño de la actividad en sí, por curiosidad o por ambición por mejorar (Gottfried, 1985; Vallerand et al., 1992). Es importante darse cuenta de que el clima del aula y la forma en que se interactúa con los estudiantes facilita o dificulta la motivación intrínseca de los alumnos. Dado que realizar cuestionarios a través de Kahoot! puede producir una sensación de diversión que provoque cierto grado de placer en los alumnos, a la par de que el alumno percibe que es una actividad que le ayuda a reforzar los conocimientos de la materia, proponemos la siguiente pregunta de investigación:

PI.3: ¿Las actividades a través de Kahoot! favorecen la motivación de los estudiantes hacia la asignatura?

Dado que, desafortunadamente, el nivel de motivación intrínseca de los estudiantes puede variar, también se debe atender a aquellos estudiantes que están menos motivados para aprender. En este sentido, la motivación extrínseca (ME) hace referencia a la participación por parte del individuo en una actividad para conseguir ciertas recompensas. En este caso, la conducta tiene significado porque está dirigida a un fin y no por sí misma (Deci y Ryan, 1985; Vallerand et al., 1992). Numerosos autores han mencionado que las recompensas extrínsecas en muchos casos estimulan la adquisición de nuevos conocimientos cuando un estudiante es bien recompensado por los logros obtenidos en clase (Ching, 2012). Dadas las características de Kahoot!, se puede establecer un sistema de recompensa definido que puede ayudar a motivar a los alumnos. Por ello, proponemos la siguiente cuestión de investigación:

PI.4. ¿Usar Kahoot! como mecanismo de recompensa en la calificación de la asignatura varía la percepción del estudiante sobre el nivel de divertimento de esta aplicación, su percepción de la utilidad como herramienta de autoevaluación, y su motivación hacia la asignatura? 


\section{Desarrollo de la innovación}

Para analizar la percepción de los alumnos sobre la herramienta Kahoot! se propusieron cuestionarios de repaso a través de esta plataforma al final de los temas impartidos en dos asignaturas de la Facultad de Ciencias Económicas y Empresariales de la Universidad de Burgos. Esta actividad se anunciaba los días previos en clase para tratar de fomentar que los alumnos repasasen la materia vista en el tema correspondiente y conseguir así, un estudio continuo de la asignatura, a la par que se trataba de fomentar la asistencia a la lección. Posteriormente, al finalizar las sesiones docentes al final del semestre, a los alumnos de ambos grupos se les realizó un cuestionario para recoger sus opiniones de manera anónima.

La muestra que utilizamos en este estudio está compuesta por dos grupos diferenciados. Así, hay que reseñar que en un grupo se premió la realización de la actividad con su grado de acierto (hasta medio punto de la evaluación final), mientras que en el otro grupo no se consideraba esta actividad dentro de la calificación de la asignatura. Este diseño muestral nos permite evaluar el efecto que tiene la existencia de una recompensa extrínseca sobre la percepción de Kahoot! y la motivación del estudiante hacia la asignatura.

La muestra final analizada en este trabajo se encuentra conformada por 116 estudiantes del Grado en Administración y Dirección de Empresas y del Grado en Finanzas y Contabilidad. De ellos, 91 (78,45\%) integran el grupo de estudiantes cuya actividad en Kahoot! se premiaba con una puntuación en la evaluación de la asignatura, mientras que los restantes 25 (21,55\%) conforman el grupo en el que la actividad en Kahoot! no se incluyó en la nota de la asignatura. La encuesta fue entregada a 5 estudiantes más (4 del primer grupo y uno del segundo) cuyas respuestas estaban incompletas, por lo que dichos cuestionarios no fueron incluidos en la muestra. Para el diseño del cuestionario nos basamos en una escala Likert de 5 puntos. Las cuestiones que se realizaron se encuentran recogidas en la Tabla 1. Como se puede apreciar, la primera pregunta mide el grado de divertimento experimentado con el empleo de Kahoot!, las tres siguientes evalúan la utilidad de esta aplicación como herramienta de autoevaluación y preparación de la asignatura, y las dos última preguntas hacen refencia a la motivación de los estudiantes hacia la asignatura, valorando el efecto que tiene Kahoot! sobre su actitud y su nivel de asistencia.

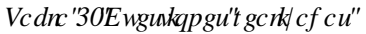

\section{Variables Pregunta realizada en el cuestionario}

Divertimento

Utilidad_Autoevaluación

Utilidad_Afianzar

Utilidad_Preparación

Actitud

Asistencia
Califique según su experiencia el grado de divertimiento que experimentó al realizar actividades basadas en el uso de Kahoot! en la asignatura.

¿Considera que Kahoot! ha sido útil como herramienta de autoevaluación para identificar el grado de conocimiento de los contenidos de la asignatura? ¿Considera que ha sido útil para afianzar sus conocimientos de la asignatura la realización de cuestionarios a través de la aplicación Kahoot!?

¿Considera que la programación de un cuestionario de Kahoot! para preparar las pruebas de evaluación en clase le ha impulsado a llevar más al día la asignatura?

¿Considera que Kahoot! ha sido una herramienta que ha favorecido su actitud hacia la asignatura?

¿Esta más incentivado a asistir a clase los días que se realiza un Kahoot! en la asignatura? 
De los 116 estudiantes que completaron el cuestionario, 54 son mujeres (46,55\% de la muestra), mientras que 62 son hombres $(53,45 \%)$. En el grupo con participación recompensada, los hombres eran mayoría $(57,14 \%)$, mientras que, en el grupo no recompensado, las mujeres participantes eran superiores en número (60\%). En promedio, los participantes tienen 20 años, siendo la media de edad prácticamente idéntica en ambos grupos (19,96 años en el grupo premiado y 20,16 años en el caso de los no recompensados).

\section{Resultados}

En la Tabla 2 se pueden observar los resultados relativos a las tres primeras preguntas de investigación planteadas anteriormente.

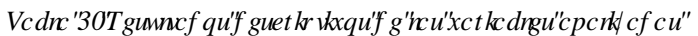

\begin{tabular}{lllllll}
\hline & Media & Mediana & Moda & Mínimo & Máximo & Desv. Est. \\
\hline Divertimento & 4,17 & 4,00 & 5 & 1 & 5 & 0,878 \\
Utilidad_Autoevaluación & 4,08 & 4,00 & 4 & 1 & 5 & 0,866 \\
Utilidad_Afianzar & 3,89 & 4,00 & 4 & 1 & 5 & 0,930 \\
Utilidad_Preparación & 3,45 & 4,00 & 4 & 1 & 5 & 1,122 \\
Actitud & 3,93 & 4,00 & 5 & 1 & 5 & 0,993 \\
Asistencia & 3,29 & 3,00 & 3 & 1 & 5 & 1,319 \\
\hline
\end{tabular}

Como se aprecia en la Tabla 2, la aplicación Kahoot! genera un elevado nivel de divertimento entre los estudiantes. Así, la respuesta más seleccionada es la que indica un mayor grado de divertimento (5), situándose la media por encima de 4. Dando respuesta a la primera de las preguntas de investigación, todo ello muestra cómo la realización de esta actividad genera entusiasmo entre la mayor parte de los estudiantes, si bien, como indica el valor mínimo, existen estudiantes a los que esta actividad no les resulta divertida.

En relación con la segunda pregunta de investigación, al analizar la percepción de utilidad de Kahoot! por los estudiantes, el valor de la mediana de las tres que se preguntan (autoevaluación, afianzar conocimiento y preparar la asignatura) se sitúa en 4. De las tres, la que obtiene una mayor valoración es la relativa a la utilidad de Kahoot! como herramienta de autoevaluación $(4,08)$, seguida de su utilidad para afianzar conocimientos $(3,89)$ y su utilidad para preparar la asignatura día a día $(3,45)$.

Respecto al incremento de la motivación por el empleo de Kahoot! (tercera pregunta de investigación), se aprecia que la actitud de los estudiantes hacia la asignatura se ve favorecida con la utilización de Kahoot! en el aula. Ello se puede inferir al observar cómo el valor más escogido es el que revela una mayor mejora en la actitud (5), situándose la media en torno a 4. En relación con la asistencia a clase los días en los que se realiza un cuestionario de Kahoot!, las respuestas se sitúan en promedio por encima del 3.

Una vez que hemos dado respuesta a las tres primeras preguntas de investigación, para responder a la cuarta de ellas, analizamos cómo la recompensa otorgada a los estudiantes en función de su grado de acierto en los cuestionarios de Kahoot! puede hacer variar la percepción de divertimento, utilidad y el incremento de la motivación interna generados por esta aplicación. 
En la Tabla 3 podemos observar los valores medios de cada una de las variables analizadas, así como la significatividad de la diferencia de dichos valores, en los dos grupos que conforman la muestra, creados en función de si los estudiantes reciben una recompensa derivada de su grado de acierto en los cuestionarios de Kahoot!. Para analizar la significatividad estadística de las diferencias de las medias de cada grupo hemos utilizado el test no pramétrico de Mann Whitney, después de comprobar que las respuestas no se distribuyen siguiendo una distribución normal (test de Kolmogorov Smirnov).

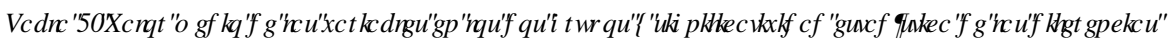

\begin{tabular}{lccc}
\hline Variable & $\begin{array}{c}\text { Grupo con } \\
\text { recompensa }(\mathbf{n}=\mathbf{9 1})\end{array}$ & $\begin{array}{c}\text { Grupo sin } \\
\text { recompensa }(\mathbf{n}=\mathbf{2 5})\end{array}$ & Sig. \\
\hline Divertimento & 4,42 & 3,28 & $* * *$ \\
Utilidad_Autoevaluación & 4,08 & 4,08 & - \\
Utilidad_Afianzar & 3,95 & 3,68 & - \\
Utilidad_Preparación & 3,51 & 3,24 & - \\
Actitud & 4,13 & 3,20 & $* * *$ \\
Asistencia & 3,44 & 2,76 & $* *$ \\
\hline
\end{tabular}

En primer lugar, la Tabla 3 muestra que la recompensa en la calificación está relacionada con una percepción de divertimento significativamente superior. Ello indica que la existencia de recompensa cambia la percepción de los estudiantes sobre el grado de divertimento de Kahoot!, quizás porque lo perciben como una forma de evaluación más dinámica y original que otras (exámenes, prácticas, trabajos, etc.).

Sin embargo, la existencia de recompensa no tiene incidencia significativa en la utilidad percibida por los estudiantes sobre el empleo de Kahoot!. Así, el hecho de que a los estudiantes se les premie su actividad en esta aplicación, no tiene relación con la percepción que los estudiantes tienen sobre Kahoot! como herramienta de autoevaluación, para afianzar conocimientos y para preparar la asignatura. Por tanto, la utilidad de Kahoot! se percibe por sus propias características, sin que la recompensa en forma de calificación incida en tal percepción.

Por último, como se observa en la Tabla 3, la existencia de recompensa sí que se encuentra relacionada con la motivación de los estudiantes. En sentido, nuestros resultados indican que el premiar la participación de los estudiantes en Kahoot! mejora la actitud de los estudiantes hacia la asignatura y fomenta la asistencia a las sesiones en las que se realizan estas actividades. Este último resultado se explica por el hecho de que la actividad en Kahoot! repercute en la calificación, por lo que los estudiantes se encuentran incentivados a asistir. Sin embargo, como hemos señalado, el empleo de esta recompensa también consigue mejorar la actitud del estudiante hacia la asignatura.

Por tanto, estos resultados muestran que la percepción sobre la utilidad de Kahoot! es independiente de la existencia de recompensas, pero, sin embargo, la percepción de divertimento, la actitud haca la asignatura y la asistencia sí que son superiores en el grupo de estudiantes en los que se les recompensa. 


\section{Conclusiones}

En este trabajo hemos analizado la percepción de estudiantes universitarios sobre la utilidad de Kahoot! como elemento dinamizador de las clases y como herramienta de autoevaluación de sus conocimientos. Asimismo, hemos estudiado la influencia de Kahoot! sobre la motivación del estudiante hacia la asignatura. Por último, también hemos analizado cómo la existencia de una recompensa por la realización de la actividad puede modificar las cuestiones previamente planteadas.

Los resultados obtenidos de la muestra analizada (116 estudiantes de la Facultad de Ciencias y Económicas de la Universidad de Burgos) revelan una percepción de divertimento elevada generada por la utilización de Kahoot!. Igualmente, los estudiantes también valoran positivamente la utilidad que tiene Kahoot! como herramienta de autoevaluación y para afianzar conocimientos estudiados en clase. Además, los estudiantes mayoritariamente creen que Kahoot! mejora su actitud hacia la asignatura y fomenta la asistencia a clase. Por último, al considerar si se incorpora o no la puntuación obtenida en los cuestionarios de Kahoot! a la calificación de la asignatura, los estudiantes del grupo en el que sí se premia la actividad en Kahoot! favorece su percepción de divertimento y la motivación de los estudiantes hacia la asignatura.

De esta manera, este estudio revela que la utilidad percibida de Kahoot! como herramienta de autoevaluación y medio para afianzar contenidos es independiente de si se recompensa la actividad. Sin embargo, el divertimento percibido sí que depende de si la actividad se premia o no. Ello puede deberse a que los estudiantes contraponen esta forma de evaluación con otras más tradicionales, con lo que, comparativamente, se incrementa ese grado de entusiasmo hacia el empleo de esta herramienta. Asimismo, como hemos señalado, la motivación de los estudiantes también mejora cuando se incorpora el sistema de recompensa. Este incremento en la motivación no se debe por tanto a que los estudiantes perciban una mayor utilidad de Kahoot!, sino a que, como acabamos de indicar, lo perciben como una forma de evaluación novedosa, original y divertida. De hecho, a los estudiantes del grupo en el que no se recompensaba la actividad se les preguntó si el introducir una recompensa mejoraría su percepción, obteniéndose un valor de 4,16 (sobre 5). Todo ello indica que la incorporación de una herramienta como Kahoot! a la docencia en titulaciones universitarias de grado mejora la motivación de los estudiantes, y, cuando además se recompensa tal actividad, dicho incremento es superior.

No obstante, este estudio cuenta con limitaciones que han de resolverse con futuras líneas de investigación. En primer lugar, la muestra podría incluir estudiantes de distintas ramas de conocimiento y diferentes cursos, para tratar de analizar si los resultados dependen de las titulaciones o del nivel de madurez de los estudiantes. Igualmente, se podrían controlar las respuestas por la calificación obtenida por los estudiantes en la asignatura, pero ello haría que dejasen de ser anónimas. En todo caso, es necesaria más investigación de cara a que la incorporación de herramientas como Kahoot! en clase se haga con unas condiciones que permitan obtener una mayor mejora en la docencia universitaria.

\section{Referencias}

BOEKAERTS, M. (2002). Motivation to learn. Brussels, Belgium: International Academy of Education.

BOYLE, T. (1997). ' HMJQIRUP XQIP HGDGODQQU. Prentice-Hall, Inc..

CHING, G. S. (2012). "Looking into the issues of rewards and punishment in students" en , QUHQDURQDO RXLQDO

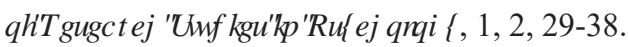

DECI, E. L. y RYAN, R. M. (1985). “The general causality orientations scale: Self-determination in personality” en - RXLQDORII5 HMDUFKIQB HURQDOW, 19, 2, 109-134. 
DECI, E. L., VALLERAND, R. J., PELLETIER, L. G. y RYAN, R. M. (1991). "Motivation and education: The selfdetermination perspective" en ( GXFDMRQDOB W FKRRJLW,26, 3-4, 325-346.

DICHEVA, D., DICHEV, C., AGRE, G. y ANGELOVA, G. (2015). "Gamification in education: A systematic mapping

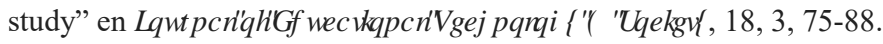

FALCHIKOV, N. y BOUD, D. (1989). “Student self-assessment in higher education: A meta-analysis” en $5 H I H Z \square R D$ ( GXFDURQDOБ HMDFK, 59, 4, 395-430.

GOTTFRIED, A. E. (1985). “Academic intrinsic motivation in elementary and junior high school students" en - RXLQDO RL( GXFDMRQDOB X FKRQRJ।, 77, 6, 631-645.

JUDSON, E. y SAWADA, D. (2002). "Learning from past and present: Electronic response systems in college lecture

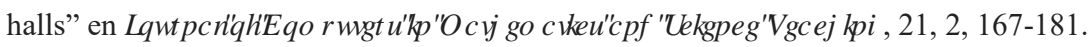

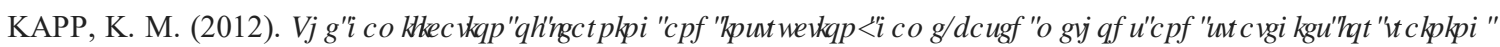
DQGHEXFDMRQ John Wiley \& Sons.

PEREZ-MANZANO, A. y ALMELA-BAEZA, J. (2018). “Gamification and transmedia for scientific promotion and for encouraging scientific careers in adolescents" en \&RP XQIFDU1O HAD( GXFDMRQ5 HMDLFL-RXLQDQ26, 55, 93-103.

PRIETO ANDREU, J. M. (2020). “Una revisión sistemática sobre gamificación, motivación y aprendizaje en universitarios" en 5HLИD, QUसХQУНHИШОUD, 32, 1, 73-99

RODRÍGUEZ, H., RESTREPO, L. \& GARCÍA, G. (2017). "Habilidades digitales y uso de teléfonos inteligentes (smartphones) en el aprendizaje en la educación superior" en 5HLWD9 IUXDOB QYYHUGDC\&DWVCFDGHD1 RUHA 50, 126142 .

VALLERAND, R. J., PELlETIER, L. G., BLAIS, M. R., BRIERE, N. M., SENECAL, C. y VALLIERES, E. F. (1992). "The Academic Motivation Scale: A measure of intrinsic, extrinsic, and amotivation in education" en ( GXFDURQDODQG SK FKRQRJIFDOP HDXUP HQW52, 4, 1003-1017.

VILLALUSTRE MARTÍNEZ, L. y DEL MORAL PÉREZ, M. E. (2015). “Gamificación: Estrategia para optimizar el proceso de aprendizaje y la adquisición de competencias en contextos universitarios” en ' LILDO GXFDRQ5 HMLZ, 27, $13-31$.

VIRVOU, M., KATSIONIS, G. y MANOS, K. (2005). “Combining software games with education: Evaluation of its educational effectiveness” en - RXLQDORIC GXFDMRQDOZHFKQRQRJ। [6RFIHW, 8, 2, 54-65.

WANG, A. I. (2015). "The wear out effect of a game-based student response system” en \&RP SXWLW प( GXFDURQ 82, 217-227.

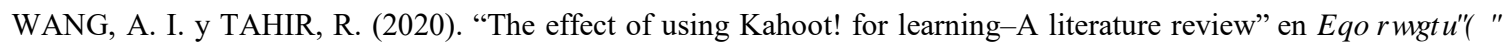
( GXFDMRQ 149, 103818.

(cc) BY-NC-ND 2021, Universitat Politècnica de València

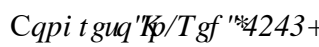

